\title{
Adaptive Emergency Braking Control With Underestimation of Friction Coefficient
}

\author{
Jingang Yi, Student Member, IEEE, Luis Alvarez, Member, IEEE, and Roberto Horowitz, Member, IEEE
}

\begin{abstract}
In this paper, a control scheme for emergency braking maneuvers in automated highway systems (AHSs) and a new online identification scheme to determine the tire-road friction characteristics of the vehicle are presented. The proposed controller determines the required pressure in the master cylinder of the braking system to achieve maximum deceleration during braking, based on the estimation of the tire-road friction characteristics and the overall braking system gain, for the given set of parameter estimates. With persistence of excitation, the identified static map between the tire longitudinal slip and the tire-road friction coefficient is guaranteed to converge to the actual map. When there is no persistence of excitation, and under a proper choice of initial conditions and adaptation gains, the proposed scheme underestimates the maximum coefficient of friction and its corresponding slip, and allows a conservative calculation of the safety critical intervehicle spacing.
\end{abstract}

Index Terms-Adaptive estimation, braking, friction, road vehicle control, vehicle.

\section{INTRODUCTION}

$\mathbf{T}$ HE CONCEPT of automated highway systems (AHSs) has been introduced to simultaneously address increasing highway capacity demands and increase traffic safety [1]. Safe feedback-based longitudinal maneuvers for AHSs and their associated control laws have been derived in [2] and [3]. These maneuvers are proven to be safe (i.e., vehicle collisions never occur) under the assumption that a lower bound on the maximum deceleration $a_{\min }$ of all vehicles is known. ${ }^{1}$ This safety critical maximum deceleration is closely related to the braking performance of a vehicle during an emergency braking maneuver, which may be needed for fault handling purposes [4]. The braking capacity of vehicles changes with adverse environmental conditions, gradual wear of components and highway topology, etc. There are two main factors that influence braking capacity: tire-road friction and available braking torque. These factors have complex behavior and the associated variables that influence this behavior are difficult to measure.

Manuscript received November 2, 2000; revised August 3, 2001. Manuscript received in final form December 27, 2001. Recommended by Associate Editor M. Jankovic. This work was supported by California PATH program under Grant MOU-312.

J. Yi and R. Horowitz are with the Department of Mechanical Engineering, University of California, Berkeley, CA 94720 USA (e-mail: jgyi@ me.berkeley.edu; horowitz@me.berkeley.edu).

L. Alvarez is with the Instituto de Ingeniería, Universidad Nacional Autónoma de México, México (e-mail: alvar@pumas.iingen.unam.mx).

Publisher Item Identifier S 1063-6536(02)03414-0.

${ }^{1}$ In addition, the maneuvers are shown to be optimal in the sense that they are completed in minimum time, while satisfying comfort and safety constraints.
In order to maintain AHS safety, it is imperative that the braking capabilities of all vehicles in the system be conservatively estimated at all times. On the other hand, to increase highway throughput it is also necessary that $a_{\min }$ be estimated as closely as possible to its actual value.

In this paper a scheme for on-line estimation of the tire-road friction coefficient of a vehicle is presented, which is guaranteed to underestimate its true value at all times, but converges to the true value under persistence of excitation conditions. A scheme for estimating an overall braking system gain is also introduced. Based on these two estimation schemes, an emergency braking controller is designed.

It is important to remark that the knowledge of the tire-road friction characteristic allows vehicles not only to adjust their spacing for safety, but this information can also be broadcast to the road-side infrastructure controller, which in turn can modify overall traffic conditions accordingly.

This paper is divided in seven sections. Section II develops a dynamic model of vehicles. Section III includes a literature review of prior tire-road friction estimation schemes and introduces the algorithm proposed in this paper. Section IV describes the design of a stabilizing controller for emergency braking while Section V describes the necessary conditions for friction underestimation. Simulation work is illustrated in Section VI. Finally, Section VII contains concluding remarks and suggests directions for future research.

\section{VEHICLE MODELING}

To describe the vehicle longitudinal dynamics, a quarter vehicle model is used. The intention of the model is dual: to develop dynamic expressions to be used later on for control purposes and to derive the coefficient of tire-road friction as a function of the vehicle dynamics. The longitudinal motion of the vehicle can be expressed by

$$
m \dot{v}=4 F_{x}-F_{a x}
$$

where $v$ is the longitudinal speed of the vehicle, $m$ is its mass, $F_{x}$ is the force at the tire and $F_{a x}$ is the aerodynamic drag force. It is assumed that forces at the tires are evenly distributed. The rotational dynamics at the wheel is described by

$$
I \dot{\omega}=\tau_{d}-\tau_{b}-F_{x} R
$$

where $\omega$ is the angular velocity, $I$ the wheel inertia, $\tau_{d}$ the driving torque, $\tau_{b}$ the braking torque and $R$ the effective rolling radius. Equations (1) and (2) assume that the longitudinal 
velocity $v$ and the wheel angular velocity $\omega$ are related through the relative velocity, $s$, defined as

$$
s=v-R \omega .
$$

During braking, relative velocity $s$ and slip $\lambda$ are related by $\lambda=s / v$. The drag force and tire force, respectively, are modeled by

$$
F_{a x}=C_{a x} v^{2} ; \quad F_{x}=-\mu F_{N}=-\mu \frac{m g}{4}
$$

with $C_{a x}$ being a properly chosen constant, $\mu$ the tire-road friction and $F_{N}=m g / 4$ the normal load in each tire.

Substituting (4) into (1) and (2) and using the time derivative of (3) yields

$$
\begin{aligned}
& \dot{v}=-c \mu-d v^{2} \\
& \dot{s}=-(a+c) \mu-b-d v^{2}+e K_{b} P_{b}
\end{aligned}
$$

with $a=R^{2} m g / 4 I, b=R \tau_{d} / I, c=g, d=C_{a x} / m$ and $e=$ $R / I$. As suggested in [5], the braking torque is approximated by $\tau_{b}=K_{b} P_{b}$, where $K_{b}$ is an overall braking system gain and $P_{b}$ the master cylinder pressure. During braking, it is assumed $\tau_{d}=0$. The velocity $v$ and relative velocity $s$ are assumed to be uniformly continuous functions of time.

\section{TIRE/ROAD FRICTION CHARACTERISTICS}

Literature for tire-road friction estimation is abundant. References [6] and [7] describe two analytical models for tire-road behavior that are intensively used by researchers in the field. In these two models the coefficient of friction, $\mu$, is mainly determined based on the wheel slip $\lambda$ and some other parameters like speed and normal load. Fig. 1 presents two curves, obtained from [8], that represent typical $\mu$ versus $\lambda$ behavior.

The expression given by [6], also known as "magic formula," was derived heuristically from experimental data to produce a good fit. It provides the tire-road coefficient of friction $\mu$ as a function of the slip $\lambda$. The expression in [7] was derived with a similar methodology. The final map expresses $\mu$ as a function of $\lambda$, the vehicle velocity, $v$, and the normal load on the tire, $F_{N}$.

Reference [9] presents a procedure for real-time estimation of $\mu$. A simplification to the analytical model by [7] is introduced in such a way that the relation between $\mu$ and $\lambda$ is linear in the parameters. Kiencke [9] used a two stages identification algorithm. In the first stage, the value of $\mu$ is estimated. This estimate of $\mu$ is used in the second stage to obtain the parameters for the simplified $\mu$ versus $\lambda$ curve.

The paper by [10] derives a scheme to identify different classes of roads. The hypothesis in [10] is that, by combining the slip and the initial slope of the $\mu$ versus $\lambda$ curve, it is possible to distinguish between different road surfaces. The author tests this hypothesis for asphalt, wet asphalt, snow, and ice and identifies the actual value of the slope with a Kalman filter and a least squares algorithm.

Reference [11] estimates $\mu$ based on a different approach. Instead of using the slip information to derive a characteristic curve, [11] estimates the forces on the tires with an extended Kalman filter. Using a tire model introduced by [12], which expresses the tire forces as a function of $\mu$, the author tries this

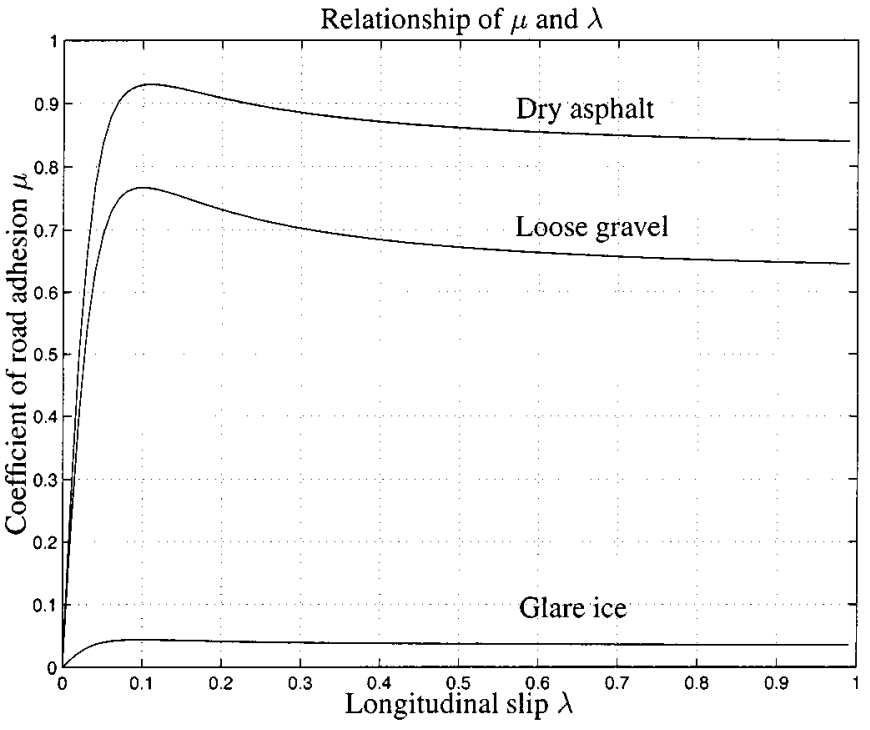

(a)

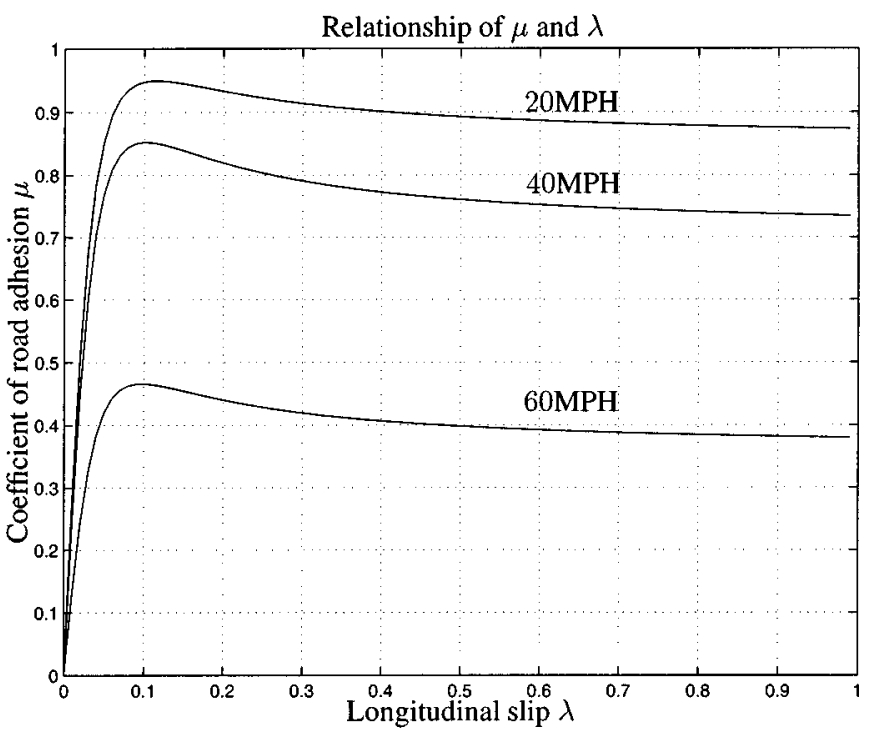

(b)

Fig. 1. Variations between coefficient of road adhesion $\mu$ and longitudinal slip $\lambda$. (a) Different road surface conditions. (b) Different vehicle velocities.

model for different values of $\mu$. A Bayesian approach is used to determine the value of $\mu$ that is most likely to produce the forces estimated with the extended Kalman filter.

The work in [9]-[11] does not consider any velocity dependence in the derivation of $\mu$, as suggested by [7] and [8]. An attempt to consider the velocity dependence for antilock braking system (ABS) control is presented in [13]. The authors assume the tire-road characteristics to be known. Due to the limitations in the available data, the authors are not able to compare their algorithm with other methods.

There are other works related to the on line identification of the tire-road friction, as for example [14] and [15]. However, in these papers only the instantaneous coefficient of friction is identified.

All the research above is based on the pseudostatic models for the road-tire friction. Recently, a LuGre dynamic model for friction was introduced in [16]. This model is applied to road-tire 
friction in [17] and [18], where lumped and distributed parameter models derived from a LuGre friction model are presented. It is shown that, with this model, it is possible to reproduce the shape of the pseudostatic magic formula. There are however some issues regarding the calibration of these models that still need further investigation. This paper is only concerned with pseudostatic friction models as the ones in [6] and [7], as the experimental data available for simulation purposes was derived with the pseudostatic approach.

For emergency braking, braking forces are large and therefore high values of slip are expected. ${ }^{2}$ If the time and distance for braking are to be minimized, emergency braking maneuvers should attempt to sustain maximum friction during all the maneuver. For this reason, it is very important to make an priori estimation of the point of maximum friction based on the information available from the instantaneous coefficient of friction, as this maximum friction point will be used as a target slip point by the emergency braking controller. The strategy in this paper is to use different points of the instantaneous coefficient of friction to identify the shape of the curve that describes the behavior of the coefficient of friction. Once this curve is identified, it is possible to deduct a proper value for the maximum coefficient of friction.

The model proposed in [7] is

$$
\mu=\left(C_{1}\left(1-e^{-C_{2} \lambda}\right)-C_{3} \lambda\right) e^{-C_{4} v}
$$

where $C_{1}, \ldots, C_{4}$ are constants and the normal load at the tire is kept constant. In this paper this model is approximated by

$$
\mu=p_{1}^{\prime} e^{-p_{2} \lambda} \lambda^{\left(p_{3} \lambda+p_{4}\right)} e^{-p_{5} v}
$$

where $p_{1}^{\prime}, p_{2}, p_{3}, p_{4}$, and $p_{5}$ are parameters to be determined. It should be noted that this parameterization is different from all previous parameterizations, including that presented in [9]. As shown in Section VI, (8) accurately approximates the behavior of (7), particularly in the region $\lambda \in\left[0, \lambda_{m}\right]$, where $\lambda_{m}$ is the point where the maximum coefficient of friction $\mu_{m}$ is attained. The structure of (8) was determined from noticing that the shape of the plot of (7) closely resembles that of $\lambda e^{\lambda}$, with the addition of other terms. Other terms and parameters in (8) were included to optimize the fit. It is convenient to point out that the model in (7) was preferred over the so-called "magic formula" as a point of departure for the approximation in (8) because of its simpler analytical structure and its closeness to the pseudostatic solution of the dynamic friction model introduced in [16].

After applying logarithm to both sides of (8) and rearranging it in vector form, we obtain

$$
y=\ln \mu=\mathbf{U} \Theta
$$

where $\mathbf{U}=[1,-\lambda, \lambda \ln \lambda, \ln \lambda,-v]$, $\boldsymbol{\Theta}=\left[p_{1}, p_{2}, p_{3}, p_{4}, p_{5}\right]^{T}$ and $p_{1}=\ln p_{1}^{\prime}$. This structure is linear in the parameters, which is a very desirable feature for the implementation parameter adaptation algorithms. An estimated $\hat{\boldsymbol{\Theta}}$ of the vector $\boldsymbol{\Theta}$ can be obtained via a standard parameter adaptation algorithm (PAA)

$$
\dot{\hat{\Theta}}=\Gamma \mathbf{U}^{T} \tilde{y}
$$

${ }^{2} \mathrm{~A}$ value of slip of $20 \%$ is considered high. where $\tilde{y}=y-\hat{y}=\mathbf{U}(\boldsymbol{\Theta}-\hat{\boldsymbol{\Theta}})=\mathbf{U} \tilde{\boldsymbol{\Theta}}, \Gamma=\Gamma^{T}>0$ is a diagonal matrix of gains and assuming that $\mu$ can be accurately measured, as discussed in Section IV.

If the velocity is kept constant, the peak value of $\mu_{m}$ can be derived from (8) as follows:

$$
\left.\mu_{m}\right|_{v=v_{0}}=p_{1}^{\prime} e^{-p_{2} \lambda_{m}} \lambda_{m}^{\left(p_{3} \lambda_{m}+p_{4}\right)} e^{-p_{5} v_{0}}
$$

where $\lambda_{m}$, the peak slip given by the solution to

$$
p_{3} \lambda_{m}\left(\ln \lambda_{m}+1\right)=p_{2} \lambda_{m}-p_{4} .
$$

Notice that although the peak friction value in (7) and (8) changes with velocity, the peak slip does not change with velocity in the same curves. This property of peak slip not depending on velocity is observed from the experimental data used to fit the pseudostatic friction models, like the "magic formula" or the Burckhardt model. Plots from experimental data are shown in Section VI.

\section{Controller Design}

In this section a controller for emergency braking that exploits the knowledge of the $\mu$ surface is designed. It is assumed that vehicles are equipped with $\mathrm{ABS}$ and that the longitudinal and angular velocities can be measured. In the case of AHS, the longitudinal velocity and acceleration can be derived from infrastructure devices designed to facilitate vehicle's position detection such as road magnets [1] and accelerometer, respectively.

Define

$$
\tilde{s}=s-s_{m}
$$

where $s_{m}=\hat{\lambda}_{m} v$ is the peak relative velocity that corresponds to the estimated peak slip $\hat{\lambda}_{m}$ at velocity $v . s_{m}$ can be obtained from (11) and (12) and the current estimation $\hat{\boldsymbol{\Theta}}$. Correspondingly, the velocity error could be defined as $\tilde{v}=v-v_{d}$, with $v_{d}$ the desired velocity; however as for emergency braking $v_{d}=0$, this definition of $\tilde{v}$ is not necessary.

The braking pressure $P_{b}$ is set as

$$
P_{b}=\frac{\hat{M}_{b}}{e}\left[(a+c) \mu+b+d v^{2}-\zeta \tilde{s}+\dot{\hat{\lambda}}_{m} v\right]
$$

where $\zeta>0$ is a gain, $\hat{M}_{b}=1 / \hat{K}_{b}$, with $\hat{K}_{b}$ the estimated value of $K_{b}$ and $\mu$ is derived from (5) under the assumption that the longitudinal acceleration can be measured. It is important to remark that the vehicle mass $m$ does not appear directly in (5), as the use of the normalized coefficient of friction $\mu$ in (4) cancels it. The drag force term, related with constant $d$ in (5), is normally small when compared with the longitudinal acceleration and does not play a critical role in emergency braking. The value of $\dot{\hat{\lambda}}_{m}$ in (14) is derived numerically.

Substituting (14) into (6) yields

$$
\dot{s}=-\zeta \tilde{s}+\dot{\hat{\lambda}}_{m} v-K_{b} \tilde{M}_{b}\left[(a+c) \mu+b+d v^{2}-\zeta \tilde{s}+\dot{\hat{\lambda}}_{m} v\right]
$$

with $\tilde{M}_{b}=M_{b}-\hat{M}_{b}$.

Define

$$
W=\frac{1}{2} \tilde{s}^{2}+\frac{1}{2} v^{2}+\frac{1}{2 \xi} K_{b} \tilde{M}_{b}^{2}+\frac{1}{2} \tilde{\boldsymbol{\Theta}}^{T} \Gamma^{-1} \tilde{\boldsymbol{\Theta}}
$$


where $\xi>0$ is a gain.

Taking the time derivative of (16) and using (6), (10) and (15) yields

$$
\begin{aligned}
\dot{W}=\tilde{s}\left\{-\zeta \tilde{s}+\dot{\hat{\lambda}}_{m} v-K_{b} \tilde{M}_{b}\right. \\
\left.\quad \cdot\left[(a+c) \mu+b+d v^{2}-\zeta \tilde{s}+\dot{\hat{\lambda}}_{m} v\right]-\hat{\lambda}_{m} \dot{v}-\dot{\hat{\lambda}}_{m} v\right\} \\
+v \dot{v}+K_{b} \tilde{M}_{b} \dot{\tilde{M}}_{b} / \xi-\tilde{\Theta}^{T} \Gamma^{-1}\left(\Gamma \mathbf{U}^{T} \tilde{y}\right) .
\end{aligned}
$$

Choose $\dot{\tilde{M}}_{b}$ as

$$
\dot{\tilde{M}}_{b}=\xi \tilde{s}\left[(a+c) \mu+b+d v^{2}-\zeta \widetilde{s}+\dot{\hat{\lambda}}_{m} v\right] .
$$

Substitute (18) in (17) to get

$$
\begin{aligned}
\dot{W} & =-\zeta \tilde{s}^{2}+\dot{v}\left(-\tilde{s} \hat{\lambda}_{m}+v\right)-\tilde{\boldsymbol{\Theta}}^{T} \mathbf{U}^{T} \mathbf{U} \tilde{\boldsymbol{\Theta}} \\
& \leq-\zeta \tilde{s}^{2}-d v^{3}\left(1+\hat{\lambda}_{m}\left(\hat{\lambda}_{m}-\lambda\right)\right)-\tilde{\boldsymbol{\Theta}}^{T} \mathbf{U}^{T} \mathbf{U} \tilde{\boldsymbol{\Theta}} \leq 0 .
\end{aligned}
$$

In the last step in (19) the facts that $\dot{v}=-c \mu-d v^{2} \leq 0$ and $v-\tilde{s} \hat{\lambda}_{m}=v\left(1+\hat{\lambda}_{m}\left(\hat{\lambda}_{m}-\lambda\right)\right)>0$ were used. Equation (19) implies that $\tilde{s}, v, \tilde{M}_{b}$ and $\tilde{\Theta}$ are bounded. Straightforward calculations show that $\ddot{W}$ is bounded. Thus, applying Barbalat's Lemma [19], it follows that

$$
\lim _{t \rightarrow \infty} \tilde{s}=\lim _{t \rightarrow \infty} v=\lim _{t \rightarrow \infty} \mathbf{U} \tilde{\boldsymbol{\Theta}}=0
$$

and therefore stability of $s=s_{m}, v=0$ follows. In addition, notice that $\tilde{y}=\ln \mu-\ln \hat{\mu}=\mathbf{U} \tilde{\Theta}$ and therefore $\lim _{t \rightarrow \infty} \mathbf{U} \tilde{\Theta}=$ $0 \Rightarrow \tilde{\mu}=0$ and that $\lim _{t \rightarrow \infty} \dot{\tilde{s}}=\lim _{t \rightarrow \infty} \dot{v}=0$. Utilizing Barbalat's Lemma again, the boundedness of $\tilde{M}_{b}$ and Eq. (15) imply that $\lim _{t \rightarrow \infty} \tilde{M}_{b}=0$. It is important to recall that only persistence of excitation will guarantee that $\lim _{t \rightarrow \infty} \tilde{\Theta}=\mathbf{0}$.

\section{FRICTION COEFFICIENT UNDERESTIMATION}

The goal in this section is to find the conditions on $\Theta$ and $\hat{\boldsymbol{\Theta}}(0)$ and the adaptation gains which will guarantee that

$$
\begin{aligned}
& \hat{\lambda}_{m} \leq \lambda_{m} \\
& \hat{\mu}_{m} \leq \mu_{m}
\end{aligned}
$$

for all time, where $\mu_{m}$ is maximum coefficient of friction and $\lambda_{m}$ the value of slip at which $\mu_{m}$ occurs. The corresponding estimated quantities are denoted as $\hat{\mu}_{m}$ and $\hat{\lambda}_{m}$, respectively. In order to derive these conditions, it is necessary to introduce some preliminary results.

Define the following function:

$$
f(\Theta, \lambda)=\frac{p_{2}}{p_{3}}-1-\ln \lambda-\frac{1}{\lambda} \frac{p_{4}}{p_{3}}
$$

that is the negative of the partial derivative in (9) with respect to $\lambda$, divided by $p_{3}$, the third component of $\Theta$. From (9) and (23), $\lambda_{m}$ and $\hat{\lambda}_{m}$ satisfy

$$
\begin{gathered}
f\left(\boldsymbol{\Theta}, \lambda_{m}\right)=0 \\
f\left(\hat{\boldsymbol{\Theta}}, \hat{\lambda}_{m}\right)=0 .
\end{gathered}
$$

Notice that (24) and (25) imply that there is always a slip point with maximum coefficient of friction for the sets of real and estimated parameters, respectively. Define

$$
\bar{\lambda}_{m}=\arg \left\{\max _{\boldsymbol{\Theta}, \lambda, v} \mu\right\}, \quad \underline{\lambda}_{m}=\arg \left\{\min _{\boldsymbol{\Theta}, \lambda, v} \mu\right\}
$$

and the interval $\Lambda_{m}=\left[0, \bar{\lambda}_{m}\right] .^{3}$

Lemma 1: If $\lambda \in \Lambda_{m}$ and $\bar{\lambda}_{m}<p_{4} / p_{3}$, then the partial derivative of (23) satisfies

$$
\frac{\partial f}{\partial \lambda} \geq 0
$$

Proof: See the Appendix.

Lemma 2: Assume Lemma 1 holds and

$$
\frac{p_{2}}{p_{3}}-\frac{\hat{p}_{2}}{\hat{p}_{3}}-\frac{1}{\bar{\lambda}_{m}}\left(\frac{p_{4}}{p_{3}}-\frac{\hat{p}_{4}}{\hat{p}_{3}}\right) \leq 0
$$

then $\hat{\lambda}_{m} \leq \lambda_{m}$.

Proof: See the Appendix.

Remark 1: The condition in (27) of Lemma 2 can be expressed in terms of the signs of $\tilde{p}_{i}, i=2,3,4$, the parameter estimation errors. For comparable sizes of $\tilde{p}_{i}, i=2,3,4$, and considering that $\bar{\lambda}_{m} \ll 1$, the choice of $\tilde{p}_{3}<0$ and $\tilde{p}_{4}>0$ is the most convenient in order for (27) to hold. Once $\tilde{p}_{3}<0$ is chosen, making $\tilde{p}_{2}<0$ follows from (27).

When the true parameters are fixed, the adaptation law in (10) can be rewritten as

$$
\dot{\tilde{\boldsymbol{\Theta}}}=-\Gamma \mathbf{U}(\lambda, v)^{T} \mathbf{U}(\lambda, v) \tilde{\boldsymbol{\Theta}}=\mathbf{A}(\lambda, v) \tilde{\boldsymbol{\Theta}} .
$$

By (20), (28) can be linearized about $\lambda=\hat{\lambda}_{m}$ and $v=0$ to obtain

$$
\dot{\tilde{\Theta}}=\mathbf{A}\left(\hat{\lambda}_{m}, 0\right) \tilde{\boldsymbol{\Theta}}+\frac{\partial \mathbf{A}(\lambda, v)}{\partial \lambda}\left(\lambda-\hat{\lambda}_{m}\right)+\frac{\partial \mathbf{A}(\lambda, v)}{\partial v} v
$$

This linearization can be further simplified if the fact that the maximum coefficient of friction is attained when $v \rightarrow 0$ is used. Thus, neglecting the velocity term in (8), it is possible to focus the analysis only in $p_{1}, \ldots, p_{4}$, the first four parameters of $\boldsymbol{\Theta}$, when analyzing the point of slip where the maximum friction occurs. If the difference $\lambda-\hat{\lambda}_{m}$ is small and $p_{5}$ is ignored, (29) can be rewritten as

$$
\dot{\tilde{\Theta}}=\mathbf{A}\left(\hat{\lambda}_{m}\right) \tilde{\boldsymbol{\Theta}}
$$

where the vector $\Theta$ and matrix $\mathbf{A}$ only consider the first four elements of $\Theta$, i.e., $p_{1}, \ldots, p_{4}$.

Solving (30), the elements of $\tilde{\boldsymbol{\Theta}}(t)$ are given by

$$
\begin{aligned}
& \tilde{p}_{1}(t)=\tilde{p}_{1}\left(t_{0}\right)-\gamma_{1} \alpha\left(t_{0}\right) f / d \\
& \tilde{p}_{2}(t)=\tilde{p}_{2}\left(t_{0}\right)+\gamma_{2} \hat{\lambda}_{m} \alpha\left(t_{0}\right) f / d \\
& \tilde{p}_{3}(t)=\tilde{p}_{3}\left(t_{0}\right)-\gamma_{3} \hat{\lambda}_{m} \ln \hat{\lambda}_{m} \alpha\left(t_{0}\right) f / d \\
& \tilde{p}_{4}(t)=\tilde{p}_{4}\left(t_{0}\right)-\gamma_{4} \ln \hat{\lambda}_{m} \alpha\left(t_{0}\right) f / d
\end{aligned}
$$


where $\gamma_{i}, i=1 . \ldots, 4$, are the elements of the diagonal matrix $\Gamma>0$,

$$
\begin{aligned}
s_{4}= & -\left(\gamma_{1}+\gamma_{2} \hat{\lambda}_{m}^{2}+\gamma_{3} \hat{\lambda}_{m}^{2} \ln ^{2} \hat{\lambda}_{m}+\gamma_{4} \ln ^{2} \hat{\lambda}_{m}\right) \\
d= & \gamma_{2} \gamma_{3} \gamma_{4}+\gamma_{1} \gamma_{2} \gamma_{3} \ln ^{2} \hat{\lambda}_{m}+\gamma_{1} \gamma_{3} \gamma_{4} \hat{\lambda}_{m}^{2} \\
& +\gamma_{1} \gamma_{2} \gamma_{4} \hat{\lambda}_{m}^{2} \ln ^{2} \hat{\lambda}_{m} \\
\alpha\left(t_{0}\right)= & \tilde{p}_{1}\left(t_{0}\right)-\hat{\lambda} \tilde{p}_{2}\left(t_{0}\right)+\hat{\lambda} \ln \hat{\lambda} \tilde{p}_{3}\left(t_{0}\right)+\ln \hat{\lambda} \tilde{p}_{4}\left(t_{0}\right) \\
f= & {\left[1-e^{s_{4}\left(t-t_{0}\right)}\right] . }
\end{aligned}
$$

Lemma 3: Assume there exists a time $t_{0}$ at which $\lambda \geq \underline{\lambda}_{m}$ and that the following conditions are satisfied:

i) $\tilde{p}_{1}\left(t_{0}\right) \geq 0, \tilde{p}_{2}\left(t_{0}\right) \leq 0, \tilde{p}_{3}\left(t_{0}\right) \leq 0, \tilde{p}_{4}\left(t_{0}\right) \geq 0$

ii) $\left|\tilde{p}_{i}\left(t_{0}\right)\right| \leq \tilde{p}_{\max }, \quad i=1, \ldots, 4$

iii) Lemma 2 holds for $t_{0}$.

Choose the gain matrix $\Gamma$ in the PAA given by (10) according to

$$
\begin{aligned}
\gamma_{1} & <\frac{\tilde{p}_{1}\left(t_{0}\right) \ln ^{2} \underline{\lambda}_{m}}{\delta} \\
\gamma_{2} & <\frac{\left|\tilde{p}_{2}\left(t_{0}\right)\right| \ln ^{2} \underline{\lambda}_{m}}{\delta \bar{\lambda}_{m}} \\
\gamma_{3} & <\frac{\left|\tilde{p}_{3}\left(t_{0}\right)\right| \ln ^{2} \underline{\lambda}_{m}}{\delta \bar{\lambda}_{m} \ln \bar{\lambda}_{m}} \\
\gamma_{4} & >1 \\
\frac{\gamma_{3}}{\gamma_{2}}\left|\ln \bar{\lambda}_{m}\right| & \geq \max \left(1, \frac{\tilde{p}_{3}(0)}{\tilde{p}_{4}(0)}\right)
\end{aligned}
$$

with $\delta=\tilde{p}_{\max }\left(1+\bar{\lambda}_{m}+\bar{\lambda}_{m} \ln \bar{\lambda}_{m}\right)$.

Then (27) in Lemma 2 is satisfied and the estimated peak value for the longitudinal slip, $\hat{\lambda}_{m}$, satisfies

$$
\hat{\lambda}_{m}(t) \leq \lambda_{m}(t), \quad \forall t \geq t_{0} .
$$

Proof: See the Appendix.

Lemma 3 defines a region in the space of parameters and $\lambda$ such that trajectories inside it, will remain in it. This region, however, does not include the case when $\lambda \approx 0$, which is expected to happen if the vehicle is cruising before an emergency braking is attempted. To analyze the effect when $\lambda$ is small, consider the following lemma.

Lemma 4: Assume that $\tilde{p}_{1}(0) \geq 0, \tilde{p}_{2}(0) \leq 0, \tilde{p}_{3}(0) \leq 0$, $\tilde{p}_{5}(0) \leq 0$ and $\tilde{p}_{2}(0) / \tilde{p}_{3}(0) \leq p_{2} / p_{3}$. By choosing $\gamma_{i}, i=$ $1,2,3,4$ as stated in Lemma 3 and in addition

$$
\gamma_{4} \geq \gamma_{4 m}
$$

where

$\gamma_{4 m}=\max \left\{-\frac{\tilde{p}_{4}(0)}{\tilde{p}_{1}(0) \ln \bar{\lambda}_{m}} \gamma_{1}, \frac{\tilde{p}_{4}(0) \bar{\lambda}_{m}}{\tilde{p}_{2}(0) \ln \bar{\lambda}_{m}} \gamma_{2}, \frac{\tilde{p}_{4}(0) \bar{\lambda}_{m}}{\tilde{p}_{3}(0)} \gamma_{3}\right\}$

then $\exists T>0$ such that the conditions in Lemma 3 are satisfied under the adaptation law for $t \geq T$ when $\lambda$ is initially small.

Proof: See the Appendix.

The previous lemmas can be summarized in the following theorem, which is our first main result.
Theorem 1: Assume that the initial conditions for the adaptation law in (10) are such that $\tilde{p}_{1} \geq 0, \tilde{p}_{2} \leq 0, \tilde{p}_{3} \leq 0$, and $\tilde{p}_{\tilde{5}} \leq 0$. Let $\gamma_{4}$ to be large relative to $\gamma_{i}, i=1,2,3$, as stated in conditions of Lemma 4 . Then after a long enough time $T$ the estimated peak value for the longitudinal slip, $\hat{\lambda}_{m}$, satisfies

$$
\hat{\lambda}_{m}(t) \leq \lambda_{m}(t), \quad \forall t \geq T
$$

Proof: See the Appendix.

Finally, the other desired result concerning the underestimation of the friction coefficient is proved in the following theorem.

Theorem 2: Assume that Lemma 3 is satisfied and in addition

$$
\tilde{p}_{1}-\tilde{p}_{5} v-\tilde{p}_{4}(1-\ln \underline{\lambda} m)-\tilde{p}_{3} \underline{\lambda}_{m}>0
$$

then the estimated peak friction, $\hat{\mu}_{m}$ satisfies

$$
\hat{\mu}_{m} \leq \mu_{m}
$$

Proof: See the Appendix.

Remark 2: The conditions of Theorem 2 are sufficient conditions for the underestimation of $\mu_{m}$. There are cases in which underestimation of $\mu_{m}$ can be achieved even when conditions in Theorem 2 are not satisfied. A more relaxed condition for underestimation of $\mu_{m}$ is that

$$
\begin{aligned}
\tilde{p}_{1}-\tilde{p}_{5} v-\tilde{p}_{4}\left(1-\ln \hat{\lambda}_{m}\right)-\tilde{p}_{3} \lambda_{m} \\
+\left(\lambda_{m}-\hat{\lambda}_{m}\right)\left(\frac{p_{4}}{\hat{\lambda}_{m}}-p_{3}\right)>0 .
\end{aligned}
$$

This condition does not depend on Lemma 3 and therefore is independent of the underestimation of $\lambda_{m}$.

Remark 3: Although conditions in Theorems 1 and 2 seem involved, the simulations results show that the important factor is to choose the signs of $\tilde{p}_{1} \geq 0, \tilde{p}_{2} \leq 0, \tilde{p}_{3} \leq 0$, and $\tilde{p}_{5} \leq 0$ when starting an emergency braking maneuver. Normally, before an emergency braking maneuver starts, the slip is small and by choosing the initial parameters and the adaptation gains to satisfy the conditions given in Lemma 4 and Theorem 2, we can always achieve the underestimation of the maximum friction coefficient and slip during the braking process even under lack of persistence of excitation. Gains can be easily tuned by trial and error so the other conditions in the theorem are satisfied. Nominal values for tires parameters obtained from tires manufacturers can be used as initial guesses of estimated parameters.

\section{Simulation RESUlTS}

Data from [20] tires \#76, 81 and 137 is used to test the approximation presented in Section III for the $\mu$ versus $\lambda$ and $v$ surface. All the test were performed under the same road conditions, same tire pressures $\left(24 \mathrm{lbf} / \mathrm{in}^{2}\right)$, same velocity $(30 \mathrm{mi} / \mathrm{h})$ and same normal forces. The nominal $\mu-\lambda$ curve for the tires are shown in Figs. 2-4.

Figs. 2-4 show that the proposed approximation in (8) fits very well the nominal formula of the road friction $\mu$ given by (7) in the region of $\left[0, \lambda_{m}\right]$ which is the region of interest. The 


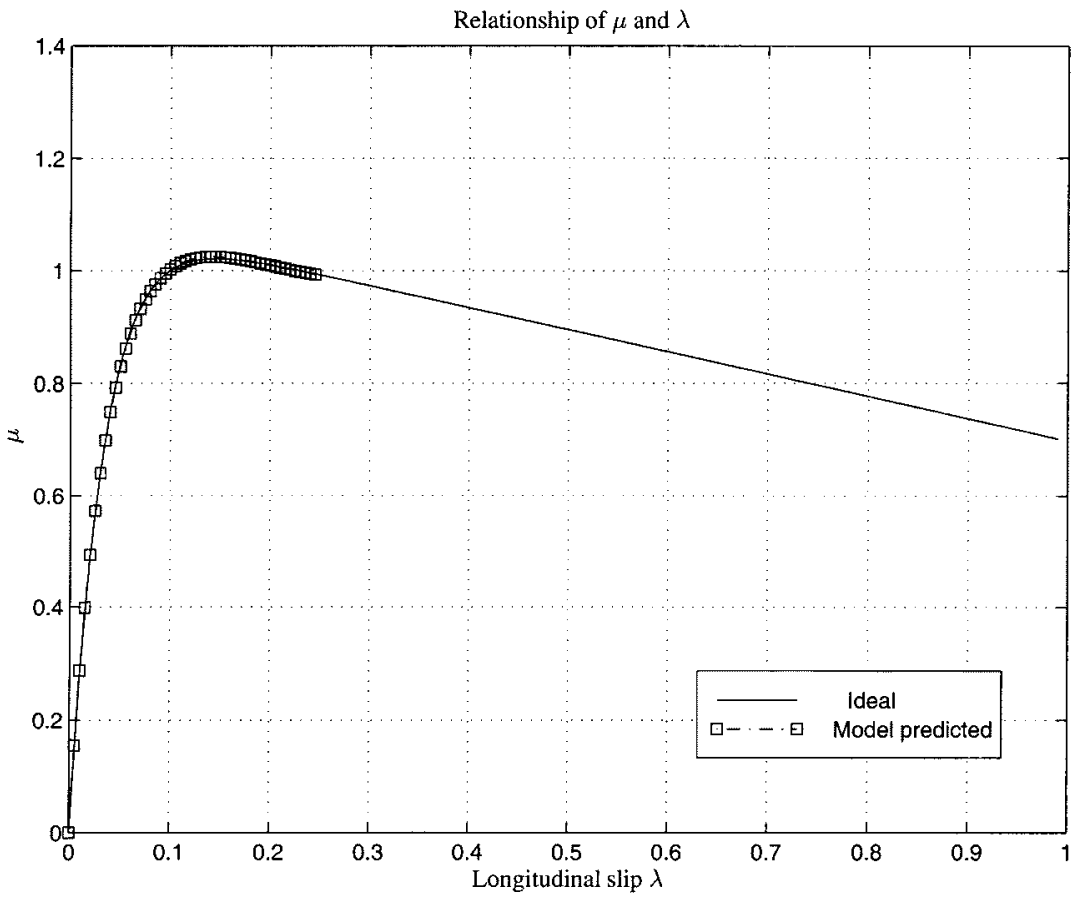

Fig. 2. Coefficients of road adhesion $\mu$ and longitudinal slip $\lambda$ by nominal and model predicted values. Tire \#76.

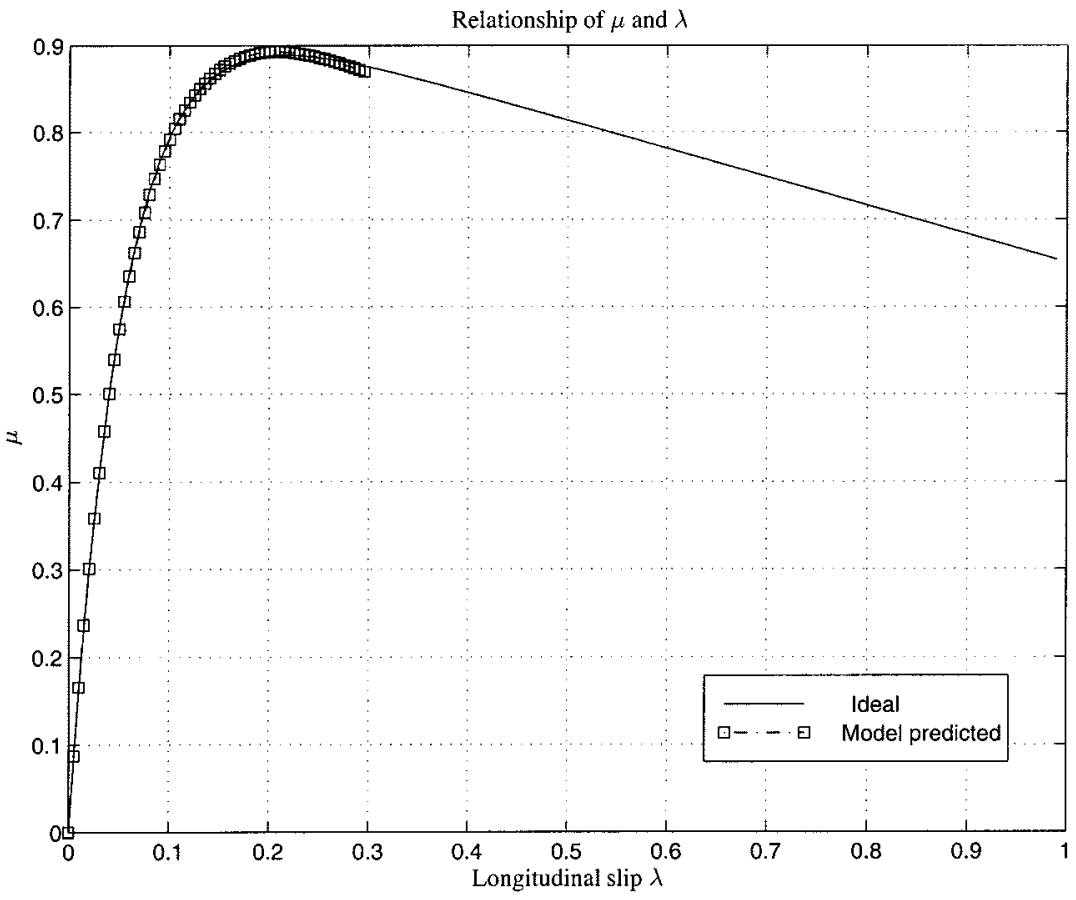

Fig. 3. Coefficients of road adhesion $\mu$ and longitudinal slip $\lambda$ by nominal and model predicted values. Tire \#81.

velocity-dependent parameter $p_{5}$ is constant since the tests in [20] were performed at constant velocity.

Adaptation of the parameters can be performed during normal driving or under emergency braking. In the first case the adapted parameters can provide a source of information to determine safe spacing policies from the vehicles in front. The simulation results presented in this section refer to a critical scenario in which there is a sudden change in the pavement characteristics that induces a change in the maximum coefficient of friction, just before and emergency braking has to be attempted. In this situation, the scheme presented in this paper achieves parameter adaptation and a close to minimal braking distance. Under this critical scenario this is close to the safest behavior one can expect.

For the simulations results of an emergency braking maneuvers using the controller introduced in the previous section the "true parameters" for the approximation in (8) were obtained with an off-line test and are shown in Table I together with the 


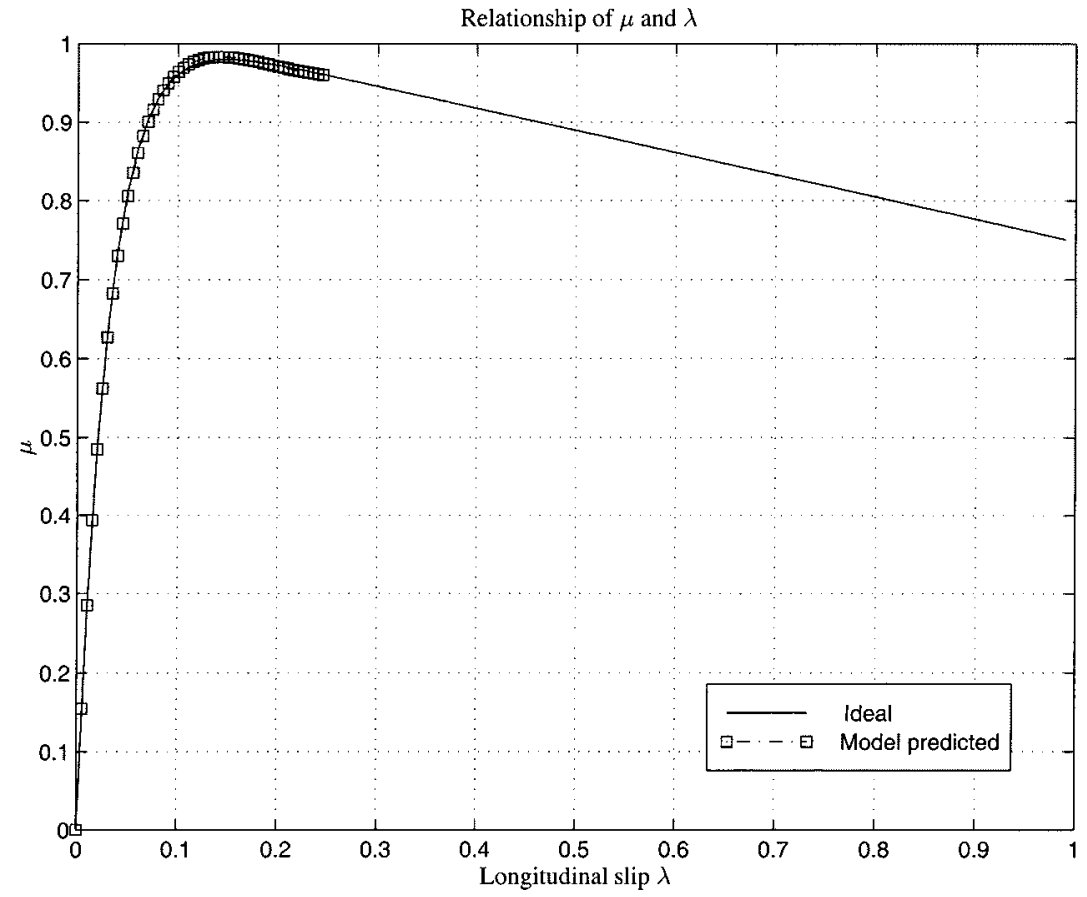

Fig. 4. Coefficients of road adhesion $\mu$ and longitudinal slip $\lambda$ by nominal and model predicted values. Tire \#137.
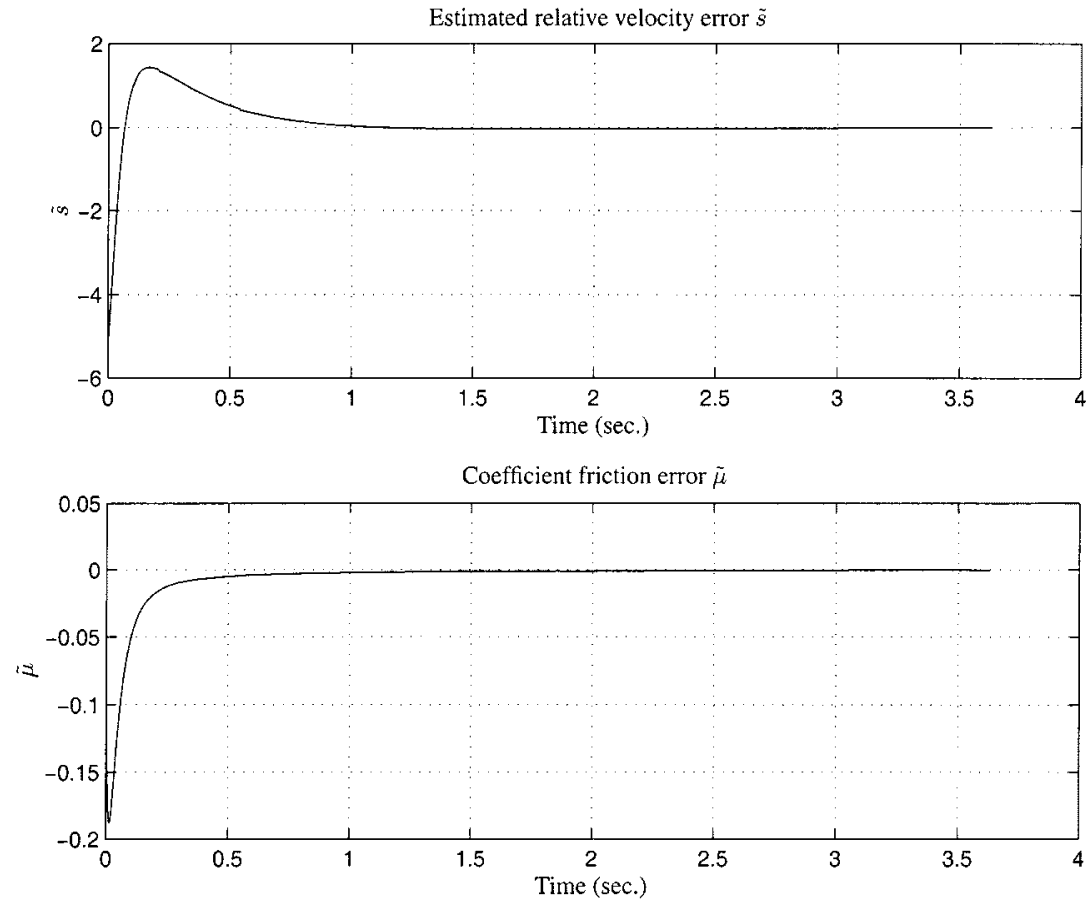

Fig. 5. Error signals.

real value of the braking system gain. Figs. 5-8 show several plots that illustrate typical simulation results for an emergency braking maneuver. Notice that in Fig. 8 parameters $\tilde{p}_{2}(t)<0$ and $\tilde{p}_{3}(t)<0$ because of the lack of excitation during the emergency braking maneuver. In this case, however, the underestimation of the maximum friction coefficient and slip are still guaranteed (Fig. 5).

Fig. 9(a) shows an example of the underestimation of the peak slip ratio when the initial values of the parameter estimates and the $p_{i}$ parameter adaptation gain satisfy the conditions of Theorems 1 and 2. Fig. 9(b) shows the case when $\tilde{p}_{1}(0)<0$ which violates one of the conditions in the Theorems. In this case underestimation of $\lambda_{m}$ does not occur.

The emergency braking maneuver was accomplished in both simulation cases, even in the case when underestimation of $\lambda_{m}$ was not achieved. This is happening because in the controller design an upper limit for the admissible value of the estimated point of maximum slip, namely $\bar{\lambda}_{m}$, was included. This limit 

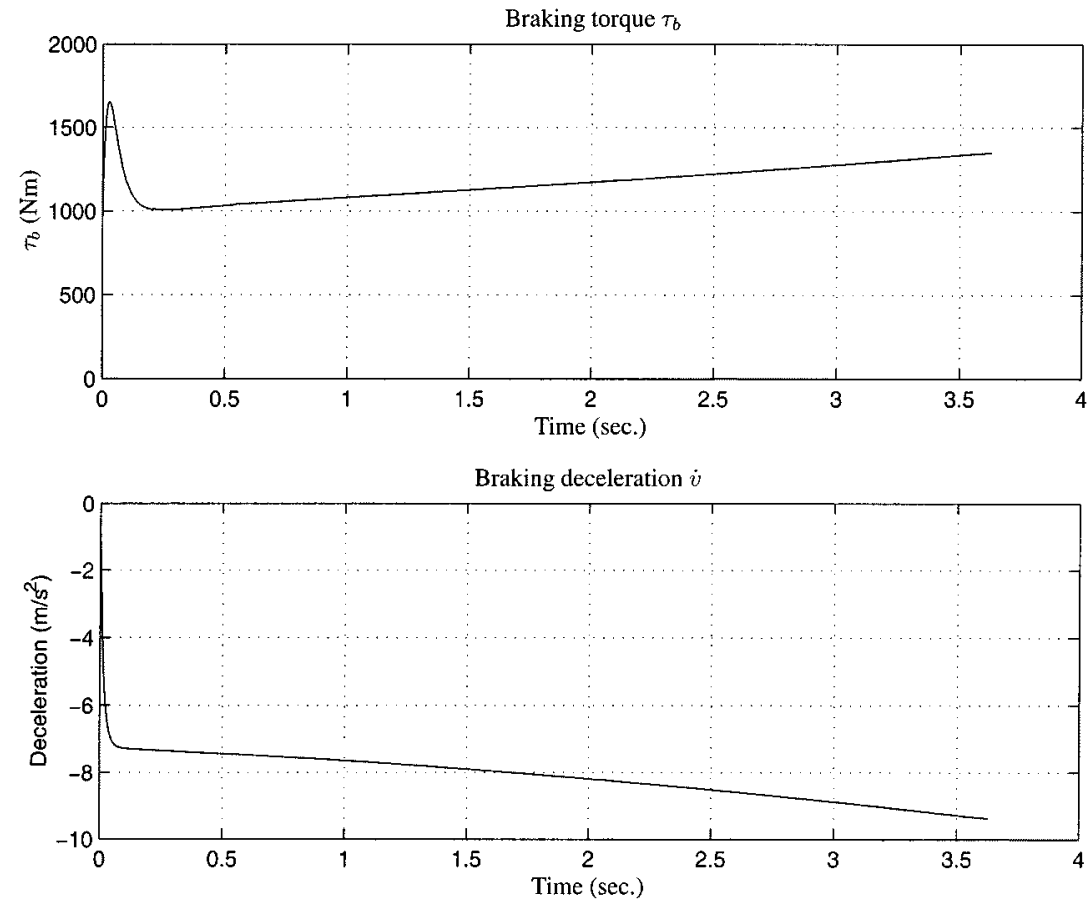

Fig. 6. Braking torque and deceleration.
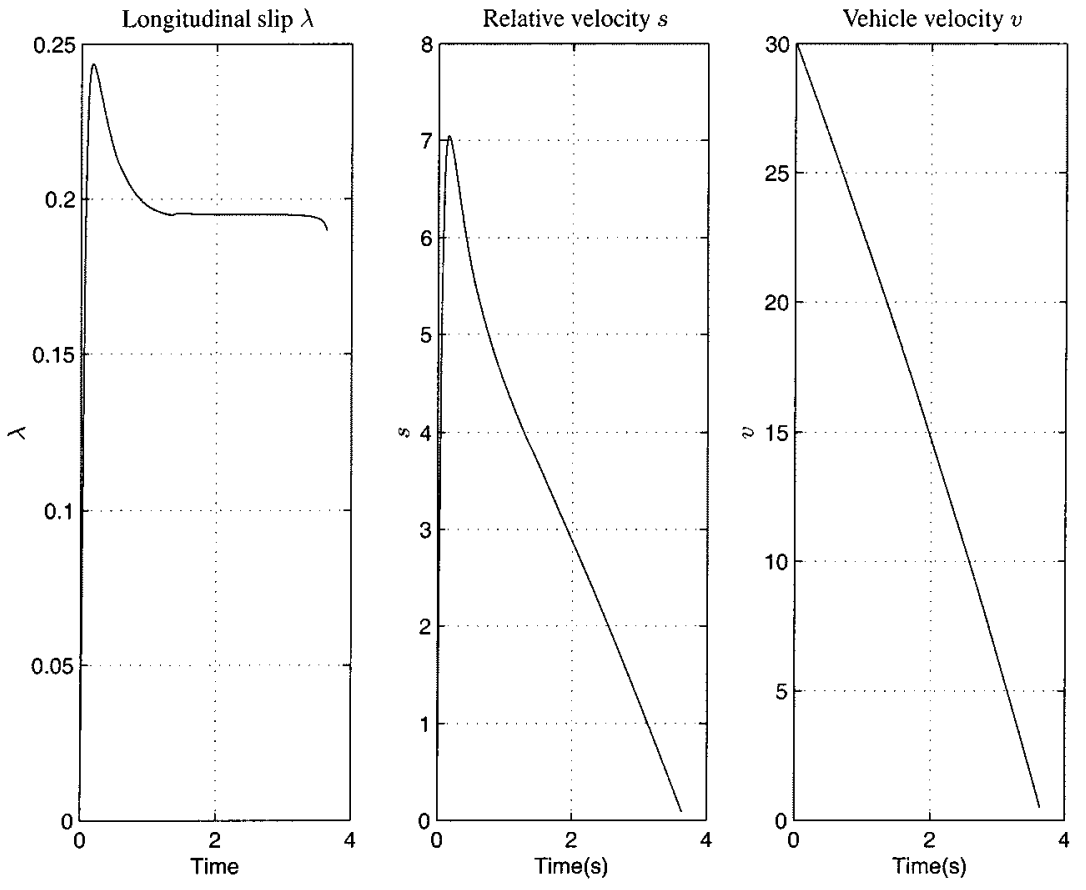

Fig. 7. Slip and state evolution versus time.

allows the emergency braking maneuver to be performed, even when the estimated friction curve is incorrect. ${ }^{4} \mathrm{~A}$ bad estimation of $\lambda_{m}$ produces, as expected, an increase in the distance that vehicles require for the emergency braking maneuver. If the tire-road friction estimation is to be used for traction control purposes other than emergency braking, the case in which underestimation is achieved [Fig. 9(a)] is clearly a good approximation to the reference tire-road friction curve and would be

${ }^{4}$ In the simulations here included, $\bar{\lambda}_{m}=0.45$ was chosen. useful for these other purposes. The other case, when underestimation is not achieved [Fig. 9(b)], is not useful for traction control purposes.

\section{CONCLUSION}

A controller for emergency braking maneuvers of vehicles equipped with ABS was designed. This controller estimates the tire-road characteristics and an overall braking system gain while trying to achieve maximum braking effort during the 

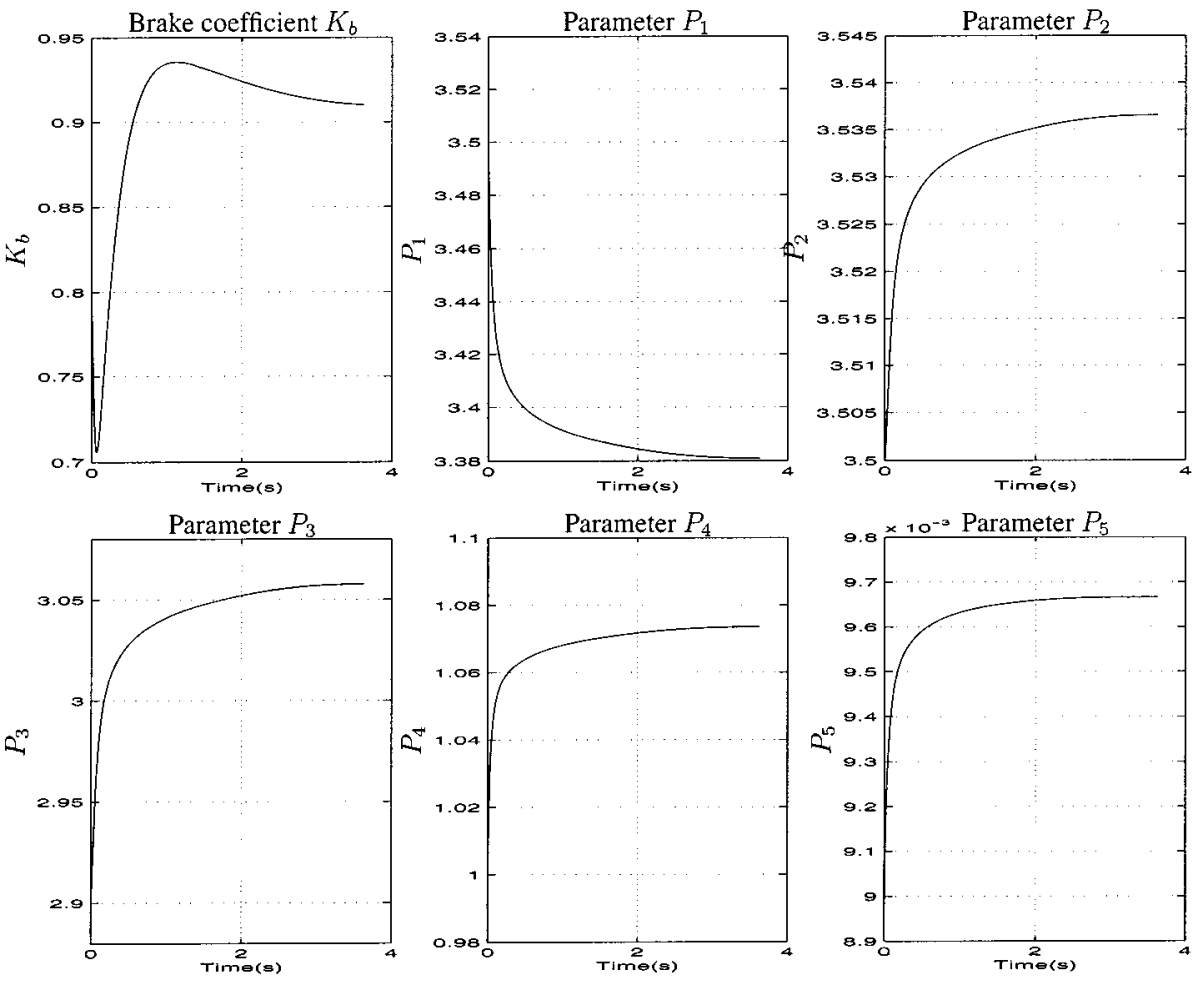

Fig. 8. Adapted parameters.

TABLE I

PARAMETERS FOR THE APPROXIMATION IN (8)

\begin{tabular}{c|c|c|c|c|c}
\hline \hline$p_{1}$ & $p_{2}$ & $p_{3}$ & $p_{4}$ & $p_{5}$ & $K_{b}$ \\
\hline 3.16 & 3.3 & 2.64 & 1.05 & 0.01 & 0.9 \\
\hline \hline
\end{tabular}

entire maneuver. Velocity dependence of the tire-road friction was explicitly considered. The stability of the controller was proven and simulation results, which are in accordance with the theoretical findings, were included. The use of this controller may provide less chattering than standard ABS controller, as this controller avoids the on-off control valve operation that is used in ABS systems for controlling the angular wheel acceleration. This controller sets a desired peak slip and avoids exceeding it in the current tire-road situation.

An important result in this paper is that, when there is not persistence of excitation and when the proper set of initial conditions and parameter adaptation gain is chosen for the estimation algorithms, the estimate of the maximum friction and the point of maximum friction, $\hat{\mu}_{m}$ and $\hat{\lambda}_{m}$, respectively are guaranteed to be smaller than or equal to the true values. Convergence to the true values is guaranteed under persistence of excitation. This is a very desirable feature for the deployment of AHS or ITS, where it is of first importance to ensure a safe operation. For this purpose of safety, the information provided with the on-line tire-road identification scheme proposed in this paper may be very useful for on-line safe spacing calculations in vehicles running under AHS or intelligent cruise control algorithms. The information can also be used by road-side infrastructure to adjust on-ramp metering control.

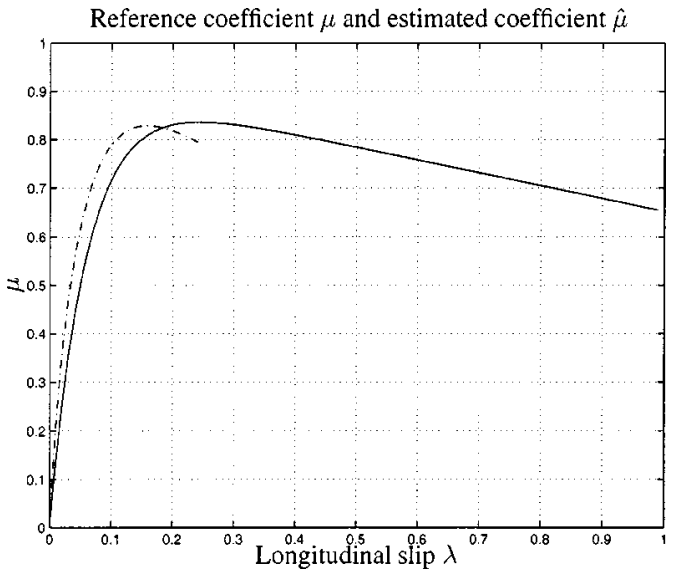

(a)

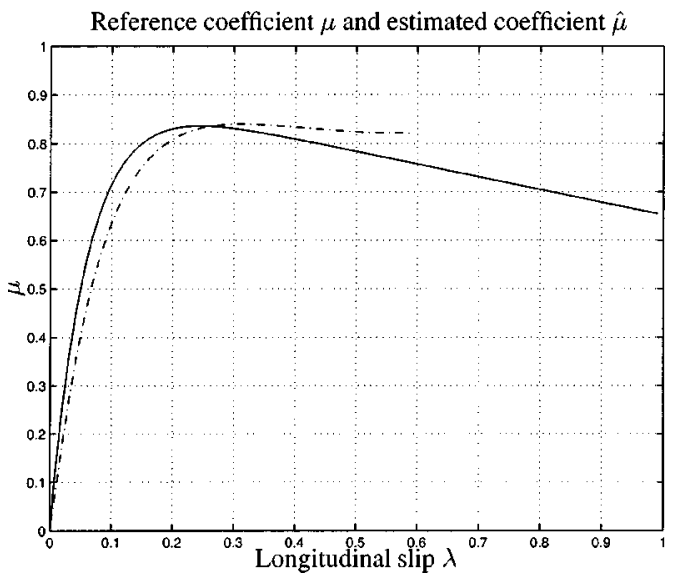

(b)

Fig. 9. Reference friction $\mu$ (solid) and estimated friction $\hat{\mu}$ (dash-dot) (a) underestimation of $\lambda_{m}$ and $\mu_{m}$; (b) no underestimation of $\lambda_{m}$. 


\section{APPENDIX}

PROOFS OF UNDERESTIMATION RESULTS

Lemma 1, Proof: The proof follows directly from the partial derivative of (23) and lemma assumptions.

Lemma 2, Proof: Equation (24) together with Lemma 1 implies that

$$
\hat{\lambda}_{m}<\lambda_{m} \Leftrightarrow f\left(\Theta, \hat{\lambda}_{m}\right)<f\left(\Theta, \lambda_{m}\right)=0 .
$$

From (23) it follows that

$$
f\left(\Theta, \hat{\lambda}_{m}\right)=\frac{p_{2}}{p_{3}}-1-\ln \hat{\lambda}_{m}-\frac{1}{\hat{\lambda}_{m}} \frac{p_{4}}{p_{3}} .
$$

Adding and subtracting the term $\hat{p}_{2} / \hat{p}_{3}$ and $1 / \hat{\lambda}_{m}\left(\hat{p}_{4} / \hat{p}_{3}\right)$ to (40) and using (25) it follows that

$$
f\left(\Theta, \hat{\lambda}_{m}\right)=\frac{p_{2}}{p_{3}}-\frac{\hat{p}_{2}}{\hat{p}_{3}}-\frac{1}{\hat{\lambda}_{m}}\left(\frac{p_{4}}{p_{3}}-\frac{\hat{p}_{4}}{\hat{p}_{3}}\right) .
$$

By (26) $\hat{\lambda}_{m} \leq \bar{\lambda}_{m}$ then

$$
f\left(\Theta, \hat{\lambda}_{m}\right) \leq \frac{p_{2}}{p_{3}}-\frac{\hat{p}_{2}}{\hat{p}_{3}}-\frac{1}{\bar{\lambda}_{m}}\left(\frac{p_{4}}{p_{3}}-\frac{\hat{p}_{4}}{\hat{p}_{3}}\right) .
$$

If condition (27) holds, $f\left(\Theta, \hat{\lambda}_{m}\right) \leq 0$ and $\hat{\lambda}_{m} \leq \lambda_{m}$.

Lemma 3, Proof: First notice that as Lemma 2 is satisfied under assumption iii) of the Lemma, then

$$
\hat{\lambda}_{m}\left(t_{0}\right) \leq \lambda_{m}\left(t_{0}\right)
$$

Using i) and ii) in Lemma assumptions, it follows that

$$
\begin{aligned}
\alpha\left(t_{0}\right) & \leq \tilde{p}_{\max }\left(1+\bar{\lambda}_{m}+\bar{\lambda}_{m} \ln \bar{\lambda}_{m}\right)=\delta \\
d & \geq \gamma_{1}+\gamma_{4} \ln ^{2} \underline{\lambda}_{m} \geq \gamma_{4} \ln ^{2} \underline{\lambda}_{m} \\
1 & \geq\left(1-e^{s} 4^{\left(t-t_{0}\right)}\right) \quad \forall t \geq t_{0} .
\end{aligned}
$$

Assume $\gamma_{4}>1$ then $d>\ln ^{2} \underline{\lambda}_{m}$, then

$$
\begin{aligned}
& \text { Eq. }(32) \Rightarrow \tilde{p}_{1}(t)>0 \quad \forall t \geq t_{0} \\
& \text { (33) } \Rightarrow \tilde{p}_{2}(t)<0 \quad \forall t \geq t_{0} \\
& \text { (34) } \Rightarrow \tilde{p}_{3}(t)<0 \quad \forall t \geq t_{0} \\
& (35) \Rightarrow \tilde{p}_{4}(t)>\tilde{p}_{4}\left(t_{0}\right)>0 \quad \forall t \geq t_{0} .
\end{aligned}
$$

Analyzing $\tilde{p}_{3}(t)$ and $\tilde{p}_{4}(t)$ in (42) it follows that

$$
-\frac{1}{\hat{\lambda}_{m}}\left(\frac{p_{4}(t)}{p_{3}(t)}-\frac{\hat{p}_{4}(t)}{\hat{p}_{3}(t)}\right)<0 .
$$

Therefore, for (27) to hold it is sufficient that

$$
\frac{p_{2}}{p_{3}}-\frac{\hat{p}_{2}\left(t_{0}\right)}{\hat{p}_{3}\left(t_{0}\right)} \geq \frac{p_{2}}{p_{3}}-\frac{\hat{p}_{2}(t)}{\hat{p}_{3}(t)} .
$$

Using (42) in (44) implies that

$$
\frac{\hat{p}_{2}\left(t_{0}\right)}{\hat{p}_{2}(t)} \geq \frac{\hat{p}_{3}\left(t_{0}\right)}{\hat{p}_{3}(t)} .
$$

For (45) to hold the ratio in the error reduction for $\tilde{p}_{2}$ has to be smaller or equal than that of $\tilde{p}_{3}$. This can be accomplished by (36).
From (36), (42), (43), and (45) it follows that condition (27) still holds for $t \geq t_{0}$ and therefore

$$
\hat{\lambda}_{m}(t) \leq \lambda_{m}(t), \quad \forall t \geq t_{0} .
$$

Lemma 4, Proof: To analyze the PAA when $\lambda$ is small consider that the adaptation law in (9) can be expressed component-wise as

$$
\begin{aligned}
& \dot{\tilde{p}}_{1}=-\gamma_{1} \tilde{y} \\
& \dot{\tilde{p}}_{2}=\gamma_{2} \lambda \tilde{y} \\
& \dot{\tilde{p}}_{3}=-\gamma_{3} \lambda \ln \lambda \tilde{y} \\
& \dot{\tilde{p}}_{4}=-\gamma_{4} \ln \lambda \tilde{y} \\
& \dot{\tilde{p}}_{5}=\gamma_{5} v \tilde{y} .
\end{aligned}
$$

The term $\tilde{y}=\mathbf{U} \tilde{\boldsymbol{\Theta}}$ is given by

$$
\tilde{y}=\tilde{p}_{1}-\tilde{p}_{2} \lambda+\tilde{p}_{3} \lambda \ln \lambda+\tilde{p}_{4} \ln \lambda-\tilde{p}_{5} v .
$$

Notice that when $\lambda \approx 0$, the term $\tilde{p}_{4} \ln \lambda$ dominates $\tilde{y}$. There are two possible cases to consider: $\tilde{p}_{4}(0) \geq 0$ and $\tilde{p}_{4}(0) \leq 0$.

Case 1: Suppose $\tilde{p}_{4}(0) \geq 0$, then $\tilde{y}(0) \approx \tilde{p}_{4}(0) \ln \lambda \leq 0$ when $\lambda$ is small. Let $t_{f}$ be the time when $\tilde{y}$ changes sign from negative to positive, i.e., $\widetilde{y}(t) \leq 0, \forall t \in\left[0, t_{f}\right]$ and $t_{i}$ be the first time parameters $\tilde{p}_{i}(t)$ change signs, respectively, for $i=$ $1,2,3,4,5$. Therefore

$$
\tilde{p}_{1}(t) \geq 0, \quad \tilde{p}_{i}(t) \leq 0, \quad i=2,3,4,5, \forall t \in\left[0, t_{f}\right] .
$$

Let $t_{\alpha}=\min \left\{t_{3}, t_{4}\right\}$ then by definition of $t_{i}$ and the facts that $\tilde{p}_{4}(0) \geq 0$ and $\tilde{p}_{3}(0) \leq 0$, we have

$$
\tilde{p}_{3}(t) \leq 0, \quad \tilde{p}_{4}(t) \geq 0, \quad \forall t \in\left[0, t_{\alpha}\right]
$$

consider $\tilde{p}_{i}(t):=p_{i}-\hat{p}_{i}(t)$. Thus, by (46)

$$
\frac{p_{4}}{p_{3}} \geq \frac{\hat{p}_{4}(t)}{\hat{p}_{3}(t)}, \quad \forall t \in\left[0, t_{\alpha}\right] .
$$

To apply Lemma 3 it is necessary to check the other conditions of Lemma 2. Note that

$$
\frac{\dot{\tilde{p}}_{3}}{\dot{\tilde{p}}_{2}}=-\frac{\gamma_{3}}{\gamma_{2}} \ln \lambda>0
$$

and then

$$
\frac{d}{d t}\left(\frac{\tilde{p}_{2}}{\tilde{p}_{3}}\right)=\frac{\tilde{p}_{2} \dot{\tilde{p}}_{2}}{\tilde{p}_{3}^{2}}\left[\frac{\tilde{p}_{3}}{\tilde{p}_{2}}-\frac{\dot{\tilde{p}}_{3}}{\dot{\tilde{p}}_{2}}\right]=\frac{\tilde{p}_{2} \dot{\tilde{p}}_{2}}{\tilde{p}_{3}^{2}}\left[\frac{\tilde{p}_{3}}{\tilde{p}_{2}}+\frac{\gamma_{3}}{\gamma_{2}} \ln \lambda\right]
$$

by the conditions in Lemma 4 it is known that $\tilde{p}_{2}(t) \leq 0$, $\dot{\tilde{p}}_{2}(t) \leq 0, \forall t \in\left[0, t_{f}\right]$, then if $\gamma_{3}$ and $\gamma_{2}$ are chosen such that

$$
\frac{\gamma_{3}}{\gamma_{2}}\left|\ln \bar{\lambda}_{m}\right| \geq \frac{\tilde{p}_{3}(0)}{\tilde{p}_{2}(0)}
$$

then

$$
\frac{d}{d t}\left(\frac{\tilde{p}_{2}}{\tilde{p}_{3}}\right) \leq 0
$$

if the initial values for $\tilde{p}_{2}(0)$ and $\tilde{p}_{3}(0)$ are chosen such that

$$
\frac{\tilde{p}_{2}(0)}{\tilde{p}_{3}(0)} \leq \frac{p_{2}}{p_{3}}
$$


then by condition (50)

$$
\frac{\tilde{p}_{2}(t)}{\tilde{p}_{3}(t)} \leq \frac{p_{2}}{p_{3}}, \quad \forall t \in\left[0, t_{f}\right]
$$

and

$$
\frac{p_{2}}{p_{3}} \leq \frac{\hat{p}_{2}(t)}{\hat{p}_{3}(t)}, \quad \forall t \in\left[0, t_{f}\right]
$$

Pick $T=\min \left\{t_{\alpha}, t_{f}\right\}$, then all conditions for Lemma 2 to hold are satisfied and thus Lemma 3 applies.

Case 2: Suppose $\tilde{p}_{4}(0) \leq 0$, then $\tilde{y}(0) \approx \tilde{p}_{4}(0) \ln \lambda \geq 0$ when $\lambda$ is small. Let $t_{f}$ be the time when $\tilde{y}$ first time changes sign and $t_{i}$ be the first time that parameters $\tilde{p}_{i}(t)$ change signs, respectively, as before in case 1 for $i=1,2,3,4,5$. Therefore

$$
\tilde{p}_{1}(t) \leq 0, \quad \tilde{p}_{i}(t) \geq 0, \quad i=2,3,4,5, \forall t \in\left[0, t_{f}\right] .
$$

Notice that for $\forall t \in\left[0, t_{f}\right]$

$$
\begin{aligned}
& \frac{\dot{\tilde{p}}_{4}(t)}{\dot{\tilde{p}}_{1}(t)}=\frac{\gamma_{4}}{\gamma_{1}} \ln \lambda<0 \\
& \frac{\dot{\tilde{p}}_{4}(t)}{\dot{\tilde{p}}_{2}(t)}=-\frac{\gamma_{4} \ln \lambda}{\gamma_{2} \lambda}>0 \\
& \frac{\dot{\tilde{p}}_{4}(t)}{\dot{\tilde{p}}_{3}(t)}=\frac{\gamma_{4}}{\gamma_{3} \lambda}>0
\end{aligned}
$$

denote $t_{f i}=\min \left\{t_{i}, t_{f}\right\}, i=1,2,3,4,5$. Similarly as in case 1

$$
\frac{d}{d t}\left(\frac{\tilde{p}_{4}}{\tilde{p}_{1}}\right)=\frac{\dot{\tilde{p}}_{1}}{\tilde{p}_{1}}\left[\frac{\dot{\tilde{p}}_{4}}{\dot{\tilde{p}}_{1}}-\frac{\tilde{p}_{4}}{\tilde{p}_{1}}\right]=\frac{\dot{\tilde{p}}_{1}}{\tilde{p}_{1}}\left[\frac{\gamma_{4}}{\gamma_{1}} \ln \lambda-\frac{\tilde{p}_{4}}{\tilde{p}_{1}}\right] .
$$

Using the facts

$$
\tilde{p}_{1}(t) \geq 0, \quad \dot{\tilde{p}}_{1}(t) \leq 0, \quad \forall t \in\left[0, t_{f 1}\right]
$$

then

$$
\frac{d}{d t}\left(\frac{\tilde{p}_{4}}{\tilde{p}_{1}}\right) \geq 0, \quad \forall t \in\left[0, t_{f 1}\right] .
$$

Noticing that $\tilde{p}_{4}(0) / \tilde{p}_{1}(0)<0$ and by picking $\gamma_{4} / \gamma_{1}$ large enough such that

$$
\frac{\gamma_{4}}{\gamma_{1}}\left|\ln \bar{\lambda}_{m}\right| \geq\left|\frac{\tilde{p}_{4}(0)}{\tilde{p}_{1}(0)}\right|
$$

then $t_{f 4} \leq t_{f 1}$, namely, $\tilde{p}_{4}(t)$ changes sign before $\tilde{p}_{1}(t)$. Similarly,

$$
\frac{d}{d t}\left(\frac{\tilde{p}_{4}}{\tilde{p}_{2}}\right)=\frac{\dot{\tilde{p}}_{2}}{\tilde{p}_{2}}\left[\frac{\dot{\tilde{p}}_{4}}{\dot{\tilde{p}}_{2}}-\frac{\tilde{p}_{4}}{\tilde{p}_{2}}\right]=\frac{\dot{\tilde{p}}_{2}}{\tilde{p}_{2}}\left[-\frac{\gamma_{4} \ln \lambda}{\gamma_{2} \lambda}-\frac{\tilde{p}_{4}}{\tilde{p}_{2}}\right] .
$$

Choosing $\gamma_{2}$ and $\gamma_{4}$ such that

$$
\frac{\gamma_{4}}{\gamma_{2}} \frac{\left|\ln \bar{\lambda}_{m}\right|}{\bar{\lambda}_{m}} \geq \frac{\tilde{p}_{4}(0)}{\tilde{p}_{2}(0)}
$$

then $t_{f 4} \leq t_{f 2}$, namely, $\tilde{p}_{4}(t)$ changes sign before $\tilde{p}_{2}(t)$. To make $t_{f 4} \leq t_{f 3}$ it is necessary that

$$
\frac{\gamma_{4}}{\gamma_{3}} \frac{1}{\bar{\lambda}_{m}} \geq \frac{\tilde{p}_{4}(0)}{\tilde{p}_{3}(0)}
$$

Thus, from the conditions (52)-(54), if (37) in Lemma 4 and (36) in Lemma 3 hold, then at $T=t_{f 4}$, by (51) and (37)

$\tilde{p}_{4}\left(t_{0}\right) \geq 0, \frac{\tilde{p}_{2}\left(t_{0}\right)}{\tilde{p}_{3}\left(t_{0}\right)} \leq \frac{p_{2}}{p_{3}}, \quad \tilde{p}_{1} \geq 0, \tilde{p}_{i} \leq 0, i=2,3,5$.

Applying Lemma 3 then it is possible to achieve

$$
\hat{\lambda}_{m}(t) \leq \lambda_{m}(t), \quad \forall t \geq T .
$$

Theorem 1, Proof: Follows directly from Lemmas 1 to 4.

Theorem 2, Proof: Proving the theorem is equivalent to show that $\mu_{m} / \hat{\mu}_{m} \geq 1$. By definition of $\mu, \hat{\mu}$ and the relationship $y=\ln \mu$, it is equivalent to show

$$
\tilde{y}_{m}:=y_{m}-\hat{y}_{m} \geq 0
$$

note that

$$
\begin{aligned}
& y_{m}=p_{1}-p_{2} \lambda_{m}+p_{3} \lambda_{m} \ln \lambda_{m}+p_{4} \ln \lambda_{m}-p_{5} v \\
& \hat{y}_{m}=\hat{p}_{1}-\hat{p}_{2} \hat{\lambda}_{m}+\hat{p}_{3} \hat{\lambda}_{m} \ln \hat{\lambda}_{m}+\hat{p}_{4} \ln \hat{\lambda}_{m}-\hat{p}_{5} v
\end{aligned}
$$

then plug above equations in (55)

$$
\begin{aligned}
\tilde{y}_{m}=\tilde{p}_{1}-\tilde{p}_{5} v-p_{2} \lambda_{m}+p_{3} \lambda_{m} \ln \lambda_{m}+p_{4} \ln \lambda_{m} \\
+\hat{p}_{2} \hat{\lambda}_{m}-\hat{p}_{3} \hat{\lambda}_{m} \ln \hat{\lambda}_{m}-\hat{p}_{4} \ln \hat{\lambda}_{m} .
\end{aligned}
$$

Recall that the slip point of maximum friction satisfies (24) and therefore

$$
p_{3} \lambda_{m} \ln \lambda_{m}-p_{2} \lambda_{m}+p_{4}=-p_{3} \lambda_{m} .
$$

Using (57) and a similar expression developed from (25) into (56) it follows that

$$
\tilde{y}_{m}=\tilde{p}_{1}-\tilde{p}_{5} v-\tilde{p}_{4}-p_{3} \lambda_{m}+p_{4} \ln \lambda_{m}+\hat{p}_{3} \hat{\lambda}_{m}-\hat{p}_{4} \ln \hat{\lambda}_{m} .
$$

Expanding $\ln \lambda_{m}$ in a Taylor series about $\hat{\lambda}_{m}$ and taking the first two terms

$$
\begin{aligned}
\tilde{y}_{m}= & \tilde{p}_{1}-\tilde{p}_{5} v-\tilde{p}_{4}-\tilde{p}_{3} \lambda_{m}-p_{3}\left(\lambda_{m}-\hat{\lambda}_{m}\right) \\
& +\tilde{p}_{4} \ln \hat{\lambda}_{m}+\frac{p_{4}}{\hat{\lambda}_{m}}\left(\lambda_{m}-\hat{\lambda}_{m}\right) \\
= & \tilde{p}_{1}-\tilde{p}_{5} v-\tilde{p}_{4}\left(1-\ln \hat{\lambda}_{m}\right)-\tilde{p}_{3} \lambda_{m} \\
& +\left(\lambda_{m}-\hat{\lambda}_{m}\right)\left(\frac{p_{4}}{\hat{\lambda}_{m}}-p_{3}\right) .
\end{aligned}
$$

By Lemma 2

$$
\left(\lambda_{m}-\hat{\lambda}_{m}\right)\left(\frac{p_{4}}{\hat{\lambda}_{m}}-p_{3}\right)>0
$$

and

$$
\begin{gathered}
-\tilde{p}_{3} \hat{\lambda}_{m}>-\tilde{p}_{3} \underline{\lambda}_{m} \\
\left|1-\ln \hat{\lambda}_{m}\right|<\left|1-\underline{\lambda}_{m}\right|
\end{gathered}
$$

and therefore for $\tilde{y}_{m}>0$ it is sufficient that

$$
\tilde{p}_{1}-\tilde{p}_{5} v-\tilde{p}_{4}\left(1-\ln \underline{\lambda}_{m}\right)-\tilde{p}_{3} \underline{\lambda}_{m}>0
$$

that is the condition required in the theorem. 


\section{REFERENCES}

[1] P. Varaiya, "Smart cars on smart roads: Problems of control," IEEE Trans. Automat. Contr., vol. 38, pp. 195-207, Feb. 1993.

[2] L. Alvarez and R. Horowitz, "Safe platooning in AHS. Part I: Safety regions design," J. Vehicle Syst. Dyn., vol. 32, no. 1, pp. 23-56, 1999.

[3] P. Li, L. Alvarez, and R. Horowitz, "AVHS safe control laws for platoon leaders," IEEE Trans. Contr. Syst. Technol., vol. 5, pp. 614-628, Nov. 1997.

[4] J. Lygeros, D. Godbole, and M. Broucke, "A fault tolerant control architecture for automated highway systems," IEEE Trans. Contr. Syst. Technol., vol. 8, pp. 205-219, Mar. 2000.

[5] J. C. Gerdes and K. Hedrick, "Brake system requirements for platooning on an automated highway," in Proc. 1995 Amer. Contr. Conf., Seattle, WA, 1995, pp. 165-169.

[6] E. Bakker, L. Nyborg, and H. Pacejka, "Tyre modeling for use in vehicle dynamic studies,", Soc. Automotive Eng. Paper \# 870 421, 1987.

[7] M. Burckhardt, Fahrwerktechnik: Radschlupf-Regelsysteme. Würtzburg, Germany: Vogel-Verlag, 1993.

[8] J. Harned, L. Johnston, and G. Scharpf, "Measurement of tire brake force characteristics as related to wheel slip (antilock) control system design," SAE Trans., vol. 8, no. 690214, pp. 909-925, 1969.

[9] U. Kiencke, "Realtime estimation of adhesion characteristic between tyres and road," in Proc. IFAC World Congr., vol. 1, 1993.

[10] F. Gustafsson, "Slip-based tire-road friction estimation," Automatica, vol. 33, no. 6, pp. 1087-1099, 1997.

[11] L. R. Ray, "Nonlinear tire force estimation and road friction identification: Simulation and experiments," Automatica, vol. 33, no. 10, pp. 1819-1833, 1997.

[12] H. T. Szostak, R. W. Allen, and T. J. Rosenthal, "Analytical modeling of driver response in crash avoidance manuevering. Volume II: An interactive tire model for driver/vehicle simulation," U.S. Dept. Transportation, Rep. DOT HS 807-271, 1988.

[13] Y. Liu and J. Sun, "Target slip tracking using gain-scheduling for antilock braking systems," in Proc. Amer. Contr. Conf., Seattle, WA, June 1995, pp. 1178-1182.

[14] H. Lee and M. Tomizuka, "Adaptive traction control," Inst. Transportation Studies, Univ. California Berkeley, PATH Res. Rep. UCB-ITS-PRR-95-32, 1995.

[15] K. Yi and T. Jeong, "Observer based estimation of tire-road friction for collision warning algorithm adaptation," JSME Int. J., vol. 41, no. 1, pp. $116-124,1998$.

[16] C. Canudas de Wit, H. Olsson, K. Åström, and P. Lischinsky, "A new model for control of systems with friction," IEEE Trans. Automat. Contr., vol. 40, pp. 419-425, Mar. 1995.

[17] C. Canudas de Wit and P. Tsiotras, "Dynamic tire friction models for vehicle traction control," in Proc. 38th IEEE Conf. Decision Contr., Phoenix, AZ, 1999.

[18] C. Canudas de Wit and R. Horowitz, "Observers for tire/road contact friction using only wheel angular velocity information," in Proc. 38th IEEE Conf. Decision Contr., Phoenix, AZ, 1999.

[19] J.-J. E. Slotine and W. Li, Applied Nonlinear Control. Engelewood Cliffs, NJ: Prentice-Hall, 1991

[20] D. J. Schuring, "Tire parameter determination," Calspan Corp., DOT HS-802 089, 1976.

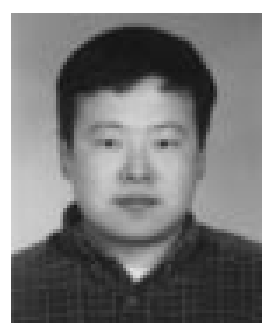

Jingang Yi (S'98) was born in Hubei Province, P.R. China. He received the B.S. degree in electrical engineering from the Zhejiang University, Hangzhou, China, in 1993, the M.Eng. degree in precision instruments from Tsinghua University, Beijing, China, in 1996, and the M.A. degree in applied mathematics from the University of California, Berkeley, in 2001, respectively. He is now pursuing the Ph.D. degree in mechanical engineering at the University of California, Berkeley.

His research interests include adaptive and nonlinear system control, intelligent transportation systems (ITS), mechatronics and computations and simulations.

Mr. Yi is an associate member of ASME.

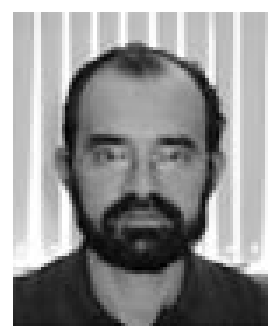

Luis Alvarez (M'00) was born in Mexico City. He received the B.S. and M.S. degrees with honors from the National Autonomous University of Mexico in 1981 and 1988, respectively. He received the Ph.D. degree from the University of California, Berkeley in 1996.

In 1987, he joined the Department of Control of the Institute of Engineering at the National Autonomous University of Mexico, where he is now Professor. His research interests include intelligent, nonlinear, and learning control with applications to vehicle and traffic control and automated highway systems.

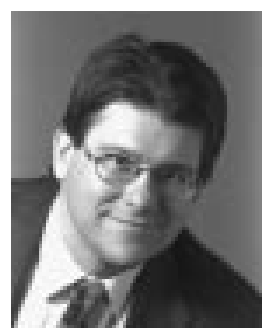

Roberto Horowitz (M'89) was born in Caracas, Venezuela, in 1955. He received the B.S. degree with highest honors in 1978 and the Ph.D. degree in mechanical engineering in 1983 from the University of California, Berkeley.

In 1982, he joined the Department of Mechanical Engineering at the University of California, Berkeley, where he is currently a Professor and Vice Chair of Graduate Studies. He teaches and conducts research in the areas of adaptive, learning, nonlinear and optimal control, with applications to microelectromechanical systems (MEMS), computer disk file systems, robotics, mechatronics and intelligent vehicle and highway systems (IVHS).

Dr. Horowitz was a recipient of a 1984 IBM Young Faculty Development Award and a 1987 National Science Foundation Presidential Young Investigator Award. He is a Member of ASME. 